\title{
Retraction Note to: Usage of COTS/EEE \\ Parts in Space Applications-An \\ Overview
}

\author{
H. V. Harish
}

Retraction Note to:

Chapter "Usage of COTS/EEE Parts in Space

Applications-An Overview" in: PSR. S. Sastry et al. (eds.),

Advances in Small Satellite Technologies, Lecture Notes in Mechanical Engineering, https://doi.org/10.1007/978-981-15-1724-2_33

The Editors have retracted this book chapter "Usage of COTS/EEE Parts in Space Applications-An Overview" because it contains material that substantially overlaps with content from a blog post by a different author [1].

All authors agree to this retraction.

[1] Dan Friedlander (2016, November 4th) COTS/EEE parts in space applications: evolution overview, revolution forward view. Intelligent Aerospace. https://www. intelligentaerospace.com/satcom/article/16538751/cotseee-parts-in-space-applicationsevolutionoverview-revolution-forward-view.

The retracted version of this chapter can be found at https://doi.org/10.1007/978-981-15-1724-2_33 